\title{
Central retinal vein occlusion after coronary artery bypass surgery: A case report
}

\author{
Ufuk Ciloglu ${ }^{1}$, Mustafa Aldag ${ }^{2}$, Sebnem Albeyoglu ${ }^{1}$, Veysel Aykut ${ }^{3}$, Canan Karakaya ${ }^{1}$, \\ Burcu Gonul ${ }^{1}$
}

${ }^{1}$ Department of Cardiovascular Surgery, Siyami Ersek Thoracic and Cardiovascular Surgery Training and Research Hospital, Istanbul, Turkey ${ }^{2}$ Department of Cardiovascular Surgery Istanbul Medeniyet University Medical Faculty, Istanbul, Turkey, ${ }^{3}$ Department of Ophthalmology, Istanbul Medeniyet University Medical Faculty, Istanbul Turkey

Correspondence: mustafa.aldag@yahoo.com

Tel.: + 905323747170

Fax.: + 902165666614

Received: 2 August 2016

Accepted: 25 March 2017

Key words: Central retinal vein occlusion - Cardiopulmonary bypass - Coronary artery bypass graft surgery.

\begin{abstract}
Objective. The aim of this study is to report a case of central retinal vein occlusion (CRVO) after coronary artery bypass graft (CABG) surgery. In this report, we present the third case in the literature with CRVO after cardiopulmonary bypass (CPB), and the first case after CABG. Case Report. A 59-year-old male patient complaining of chest pain was admitted to our hospital. The patient underwent an elective coronary angiography and was diagnosed with three-vessel coronary artery disease. An uneventful coronary artery bypass graft operation was performed using $\mathrm{CPB}$. On the second postoperative day, the patient described blurring and decreased vision in his left eye, whereas the right eye was normal. The anterior-posterior segment was examined by an ophthalmologist who diagnosed central retinal vein occlusion, using a visual acuity test, fundoscopy. After 5 months of treatment, there has been no improvement in the patient's visual acuity. Conclusion. As two previous case reports indicated CRVO can be a rare complication after $\mathrm{CPB}$, this study demonstrated that $\mathrm{CRVO}$ can also be a complication of CABG. Therefore, CRVO should always be considered as a potential complication after cardiac surgery.
\end{abstract}

\section{Introduction}

Central retinal vein occlusion (CRVO) is a retinal vascular disorder and a cause of painless visual loss, which is the second most common cause of decreased visual acuity after diabetic retinopathy. The clinical manifestations of CRVO are related to venous congestion, which leads to optic disc swelling, widespread intra-retinal hemorrhage, macular edema, and ischemia (1). Macular edema is the most common cause of decreased vision in patients with CRVO (2). Risk factors for CRVO found in previous clinical and epidemiologic studies include: age, arterial hypertension, diabetes mellitus, glaucoma, high intraocular pressure, and acquired or congenital coagulopathies (Homocysteinemia, anticardiolipin and antiphospholipid syndromes, factor V Leiden, protein $\mathrm{S}$ and protein $\mathrm{C}$ mutations). Some studies also have reported coronary artery disease and kidney disease as risk factors (3, 4). The exact pathogenesis of the thrombotic occlusion of the central retinal vein is not known. Various local and systemic factors play a role in the pathological closure of the central retinal vein. 
Cardiopulmonary bypass during cardiac surgery is an important procedure which effects coagulation. There are some complications of cardiopulmonary bypass resulting from impaired coagulation cascade, including cerebrovascular accidents, peripheral embolism, renal vein thrombosis and CRVO. The relationship between cardiac surgery and central retinal vein occlusion has been previously documented. Fosnat et al. (5) reported a patient who developed CRVO immediately following extensive cardiac surgery including aortic valve, aortic root, and proximal aortic arch replacements, as well as coronary artery bypass grafts. Also, no certain method has been found so far to treat this morbidity.

In this diagnostic case report, we present the third case of CRVO after cardiopulmonary bypass, and the first one after coronary artery bypass grafting (CABG) surgery.

\section{Case report}

A 59-year-old male patient complaining of chest pain was admitted to our hospital. He was under medication for hypertension and type II diabetes mellitus. His blood pressure and blood glucose levels were within normal ranges. A treadmill stress test was carried out. Chest pain and ST segment changes

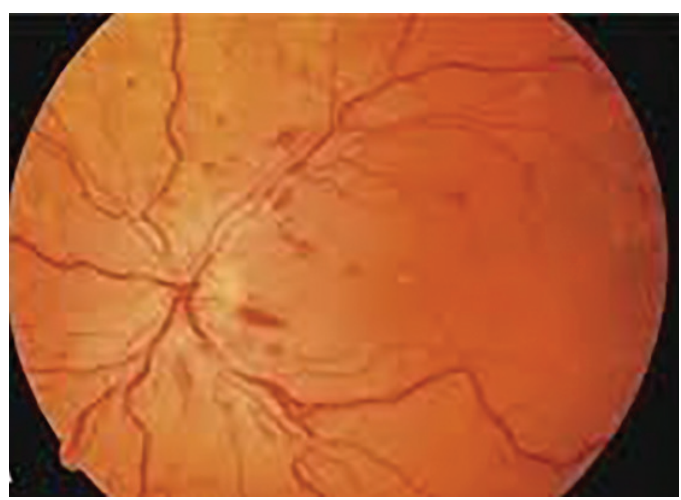

Figure 1 The dilated fundus examination; retinal hemorrhages throughout the four quadrants, as well as disc edema and dilated, tortuous retinal veins. were determined by the ECG, so the test was ended. Cardiac enzyme tests were negative. The patient underwent elective coronary angiography and was diagnosed with three-vessel coronary artery disease. We performed coronary artery bypass grafting surgery, including left anterior, circumflex and right coronary arteries. Massive bleeding was observed from chest tubes (1500 cc in total) in the early postoperative hours, so we operated on the patient again to locate the source, but no surgical bleeding spot was detected. In the meantime, we transfused two units of fresh blood, erythrocyte suspension and fresh frozen plasma. After the second intervention, no significant bleeding was observed and the patient was extubated on the same day.

On the second postoperative day, the patient described blurring and decreased vision in his left eye. The patient was then examined by an ophthalmologist and on examination the best corrected visual acuity was logMAR 1.0 (OD) and 0.8 logMAR (OS). Anterior segment examination revealed a clear cornea with grade one nuclear sclerosis in both eyes. The intraocular pressure was measured to be $14 \mathrm{mmHg}(\mathrm{OD})$ and 17 $\mathrm{mmHg}$ (OS) by Goldmann applanation tonometry. The pupils were normal in size and reaction to light and accommodation. Posterior segment examination revealed clear media with normal sized optic disc and a Cup: Disc ratio of 0.3 in both eyes. The vessels were dilated and tortuous with multiple flame-shaped and dot-blot hemorrhages in all four quadrants of left eyes (Figure 1). The patient was given anti-inflammatory agent (nepefenac $0.1 \%$ ) to resolve macular edema and low-molecular-weight-heparin (enoxaparin) to provide anticoagulation (6). After 5 months of treatment, there has been no improvement in the patient's visual acuity. 


\section{Discussion}

Central retinal vein occlusion takes the second place (after diabetic retinopathy) of all retinal vascular diseases causing loss of visual acuity (7). Some risk factors for CRVO have been reported, including age, smoking, systemic hypertension, diabetes mellitus, open angle glaucoma and decreased physical activity, but the exact pathology remains unknown. Various local and systemic factors play a role in the pathological closure of the central retinal vein, including compression of the vein (mechanical pressure due to structural changes in lamina cribrosa, eg, glaucomatous cupping, inflammatory swelling in optic nerve, orbital disorders); hemodynamic disturbances (associated with hyperdynamic or abnormal circulation); vasculitis; homocystinuria, anticardiolipin and antiphospholipid syndromes, factor $\mathrm{V}$ Leiden, protein $\mathrm{S}$, protein $\mathrm{C}$, and prothrombin G20210A mutations. Besides these clinical data, other pathophysiological elements that effect coagulation cascade may cause clotting, resulting in vein occlusion. These are; platelet activity, aggregation of red blood cells, fibrinogen concentration, and haematocrit levels $(8,9)$.

CRVO may be observed as two clinically important subtypes. The first is non-ischemic CRVO, which results from venous stasis in the retina, accounting for about 75\% of all CRVO cases. The second is ischemic CRVO, which results from venous occlusion. The distinction between these two types is significant, due to their different treatment and prognosis. Ischemic CRVO was defined as severe visual loss (6/60 or less), extensive retinal hemorrhages and cotton-wool spots, and poor perfusion to the retina as observed in fleuroscein angiography, which is the more severe and potentially blinding type. The non-ischemic type may advance to the ischemic type (10). Klein et al. reported the incidence of CRVO in the normal popula- tion to be $0.5 \%$ in their 15 -year follow-up study of 2119 people (11).

Although there is no certain treatment, ophthalmologists are currently using several drugs in management of CRVO, such as intravitreal injections of anti-vascular endothelial growth factor (anti-VEGF) agents or steroids (12). Also anti-inflammatory agents do not form part of the standard treatment of retinal vein occlusion. Anti-VEGF and intravitreal steroid injections are not aimed at treating the CRVO, but the macular oedema secondary to CRVO.

The patient was a 59-year-old male, a smoker (20 packs/year). He also underwent a cardiopulmonary bypass, which may have caused the low coagulation factor levels and low platelet counts that resulted in major bleeding followed by massive transfusion of blood products, postoperatively. In our opinion, CRVO occurred due to the first surgery (due to use of $\mathrm{CPB}$ ). As well as bleeding diathesis and transfusions there were other predisposing factors for the development of CRVO. Use of agents such as protamine or tranexamic acid to prevent bleeding may provoke thrombosis and cause alterations in coagulation (13). Regarding the selection of treatment of CRVO, macular edema is one of the prominent treatable causes of decreased visual acuity in patients with CRVO. Various treatment modalities have been used for different components of macular edema pathogenesis, with significant progress in stabilizing or improving visual acuity. The suggested treatments are as follows: anti-inflammatory agents; intravitreal injection of ranibizumab, aflibercept, triamcinolone and bevacizumab. Pharmacological treatment with intravitreal anti-vascular endothelial growth factor (VEGF) agents is currently first-line therapy for macular edema. We used low-molecular-weight-heparin (enoxaparin) in the treatment of this patient, according to the ophtalmologist's suggestions. Also, a topical non-steroidal anti-inflammatory agent 
(nepafenac $0.1 \%$ ) was administered, to reduce macular edema until the venous occlusion resolved (6). The patient's macular edema was minimally cystoid so no other treatments were considered. Moreover, intravitreal anti-VEGF Injection increases risk of heart attack in coronary heart patients (14). There was no improvement, and the patient had not regained full vision in his left eye after 5 months of treatment.

\section{Conclusion}

CRVO following cardiopulmonary bypass is very rare. As two previous case reports indicated that CRVO can be a complication after $\mathrm{CPB}$, this study demonstrated that CRVO can also be a complication after CABG (15). Therefore, CRVO should always be considered as a potential complication after cardiac surgery.

\section{What is already known on this topic}

Central retinal vein occlusion is a a common cause of vision impairment and a cause of painless visual loss, and is the second most common cause of decreased visual acuity after diabetic retinopathy. The relationship between cardiac surgery and central retinal vein occlusion has been previously documented.

\section{What this study adds}

In the literature there are only two known cases of central retinal vein occlusion after cardiopulmonary bypass. This study presents the third case presented in the literature after cardiopulmonary bypass and the first after coronary bypass grafting surgery.

Authors' contributions: Conception and design: UC, MA and CK; Acquisition, analysis and interpretation of data: SA, CK and BG; Drafing the article: MA, CK and BG; Revising it critically for important intellectual content: UC and SA; Approved final version of the manuscript: UC, MA and BG.

Conflict of interest: The authors declare that they have no conflict of interest.

\section{References}

1. Bertelsen M, Linneberg A, Christoffersen N, Vorum $\mathrm{H}$, Gade E, Larsen M. Mortality in patients with central retinal vein occlusion. Ophthalmology. 2014;121(3):637-42.

2. Heier JS, Clark WL, Boyer DS, Brown DM, Vitti R, Berliner AJ, et al. Intravitreal aflibercept injection for macular edema due to central retinal vein occlusion: two-year results from the COPERNICUS study. Ophthalmology. 2014;121(7):1414-20.

3. Lim LL, Cheung N, Wang JJ, Islam FM, Mitchell $\mathrm{P}$, Saw SM, et al. Prevalence and risk factors of retinal vein occlusion in an Asian population. $\mathrm{Br} \mathrm{J}$ Ophthalmol. 2008;92(10):1316-9.

4. Shahsuvaryan ML, Melkonyan AK. Central retinal vein occlusion risk profile: a case-control study. Eur J Ophthalmol. 2003;13(5):445-52.

5. Fosnot J, Glazer-Hockstein C, Tolentino MJ. Central retinal vein occlusion immediately following cardiac surgery. Ophthalmic Surg Lasers Imaging. 2003;34(3):215-6.

6. Hariprasad SM, Akduman L, Clever JA, Ober M, Recchia FM, Mieler WF. Treatment of cystoid macular edema with the new-generation NSAID nepafenac 0.1\%. Clin Ophthalmol. 2009;3:147-54.

7. Brand CS. Management of retinal vascular diseases: a patient-centric approach. Eye (Lond). 2012;26 Suppl 2:S1-16.

8. Risk factors for central retinal vein occlusion. The Eye Disease Case-Control Study Group. Arch Ophthalmol. 1996;114(5):545-54.

9. Michalska MK, Spiewak D, Slowinska LL, Sierocka SJ. Influence of hemorheological factors on the development of retinal vein occlusion. Clin Hemorheol Microcirc. 2016;63(1):69-76.

10. Hayreh SS. Classification of central retinal vein occlusion. Ophthalmology. 1983;90(5):458-74.

11. Klein R, Moss SE, Meuer SM, Klein BE. The 15year cumulative incidence of retinal vein occlusion: The Beaver Dam Eye Study. Arch Ophthalmol. 2008;126(4):513-8.

12. Steigerwalt RD, Pascarella A, De Angelis M, Ciucci F, Gaudenzi F. An ultra-low-molecular-weight heparin, fondaparinux, to treat retinal vein occlusion. Drug Discov Ther. 2016;10(3):167-71.

13. Gelb AB, Roth R, Levin J, London MJ, Noall RA, Hauck WW, et al. Changes in blood coagulation during and following cardiopulmonary bypass: lack of correlation with clinical bleeding. Am J Clin Pathol. 1996;106(1):87-99.

14. Ng WY, Tan GS, Ong PG, Cheng CY, Cheung CY, Wong DW, et al. Incidence of myocardial infarction, stroke, and death in patients with age-related macular degeneration treated with intravitreal anti-vascular endothelial growth factor therapy. Am J Ophthalmol. 2015;159(3):557-64.e1.

15. Allinson RW, Limstrom SA, Sethi GK, Copeland JG. Central retinal vein occlusion after heart-lung transplantation. Ann Ophthalmol. 1993;25(2):5863. 